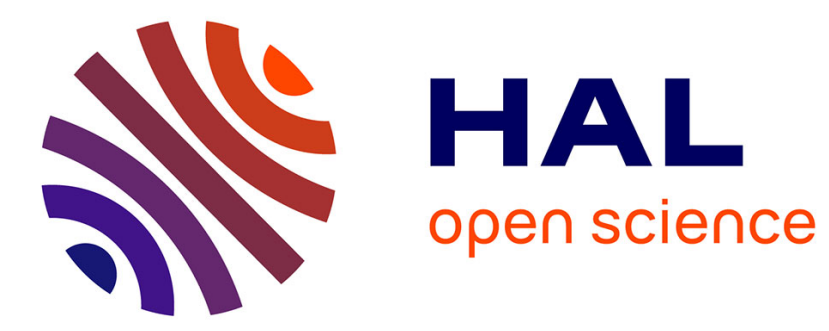

\title{
Babinet-bilayered geometric phase optical elements
}

Etienne Brasselet

\section{To cite this version:}

Etienne Brasselet. Babinet-bilayered geometric phase optical elements. Optics Letters, 2018, 43 (11), pp.2623-2626. 10.1364/OL.43.002623 . hal-01802762

\section{HAL Id: hal-01802762 \\ https://hal.science/hal-01802762}

Submitted on 29 May 2018

HAL is a multi-disciplinary open access archive for the deposit and dissemination of scientific research documents, whether they are published or not. The documents may come from teaching and research institutions in France or abroad, or from public or private research centers.
L'archive ouverte pluridisciplinaire HAL, est destinée au dépôt et à la diffusion de documents scientifiques de niveau recherche, publiés ou non, émanant des établissements d'enseignement et de recherche français ou étrangers, des laboratoires publics ou privés.

\section{(이) $\$$}

Distributed under a Creative Commons Attribution - NonCommercial - NoDerivatives| 4.0 


\title{
Babinet-bilayered geometric phase optical elements
}

\author{
Etienne Brasselet \\ Univ. Bordeaux, CNRS, LOMA, UMR 5798, F-33400 Talence, France (etienne.brasselet@u-bordeaux.fr)
}

\begin{abstract}
Geometric phase optical elements based on structured anisotropy are widely used for phase shaping via their orientational degree of freedom. To date, amplitude shaping via space-variant retardance is much less investigated, a practical reason being that the spin-orbit interaction of light couples retardance with the dynamic part of the optical phase. Inspired by the complementary diffractive elements associated with Babinet's principle, a bilayered subwavelength grating design is proposed in order to cancel out the spatial modulation of the dynamic phase usually associated with space-variant birefringent phase retardation. This concept is illustrated in the framework of single-mode Laguerre Gauss -beam shaping.
\end{abstract}

OCIS codes: (050.6624) Subwavelength structures; (140.3300) Laser beam shaping.

Structuring matter at the subwavelength scale is known to allow the design and manufacture of artificial materials with wave properties that are not possessed by the constituting bulk media taken apart. These materials are usually called metamaterials, a name derived from the Greek "meta" (beyond) emphasizing the access to novel properties and phenomena that seems limited so far only by our imagination. In electromagnetism, metamate rials evolved from radio frequencies to optics with substantial conceptual and engineering advances during the last two dec ades [1]. This concept went beyond electromagnetism, and has been addressed when thermodynamics, acoustics, elasticity, or quantum mechanics are at play [2]. Quite naturally, the process of progress has led to the development of dynamic versions of static metamaterials toward the creation of enhanced or novel functionalities [3,4] and now embarks the richness and subtleties of nonlinear physics as well $[5,6]$.

Of course, advanced designs continue to be developed even in the linear case. For instance, in the context of spin controlled asymmetric orbital angular momentum states, it has been pro posed recently to encode independent dynamic and geometric phase profiles into single metallic [7] or dielectric [8] metasur faces. In practice, this is made by controlling locally the orien tation and aspect ratio of equally spaced subwavelength anisotropic nanostructures. Besides phase control, amplitude shaping is also accessible to metasurfaces by exploiting their vectorial nature. This requires the combination of the local con trol of the birefringent phase retardation with post polarization filtering, which has been exploited recently in the context of modal beam shaping [9]. In short, the optical characteristics of a passive metasurface are determined by the spatial distribu tion of three quantities: (i) the dynamic phase, $\Phi_{\text {dyn }}$, which is associated with the average optical path length; (ii) the geomet ric phase, $\Phi_{\text {geom }}$, which uniquely depends on the structure of the medium; and (iii) the birefringent phase retardation, $\Delta$, which arises from anisotropic phase delay between the ordinary and extraordinary waves. From a general point of view, $\Phi_{\mathrm{dyn}}$, $\Phi_{\text {geom }}$, and $\Delta$ can be independently adjusted by design. However, this requires a conception step that relies on optimiz ing these parameters via numerical simulations, which prevents from an intuitive approach to conceive novel photonic functionalities.

Here, inspired by the Babinet's principle, a bilayered design made of two complementary subwavelength gratings (see Fig. 1) is proposed with the aim at getting rid of the dynamic phase spatial modulation usually associated with amplitude modulation driven by space variant birefringence. This is done by multiplexing two independent structural degrees of freedom that independently act on amplitude and phase. Namely, we

(a) Homogeneous layer

(b) Babinet bilayer

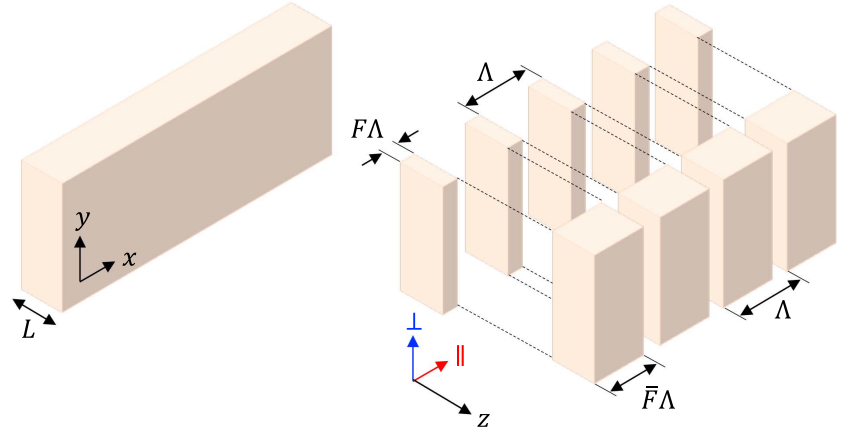

Fig. 1. (a) Slab of an isotropic dielectric with thickness $L$. (b) Proposed Babinet bilayered design that consists of two comple mentary subwavelength gratings made by splitting the slab shown in panel (a). By construction, the two complementary gratings have identical periods, $\Lambda$, and complementary filling factors, $F$ and $F=1 \quad F$. 
address the design of metasurfaces having a uniform $\Phi_{\text {dyn }}$ while (i) the grating period and its filling factor define $\Delta$, and (ii) the orientation angle, $\psi$, of the grating wavevector defines $\Phi_{\text {geom }}=2 \sigma \psi$, where $\sigma= \pm 1$ is the helicity of the incident light. In order to gain insight, it is worth recalling that the behavior of a transparent optical metasurface in the paraxial regime can be grasped simply and analytically. Indeed, by con sidering an incident circularly polarized field described by the unit vector $\mathbf{c}_{\sigma}=(\mathbf{x}+i \sigma \mathbf{y}) / \sqrt{ } 2$, where $(x, y)$ is the plane of the metasurface, the output light field can be expressed as [10]

$$
\mathbf{E}_{\text {out }} \propto e^{i \Phi_{\mathrm{dyn}}}\left[\cos (\Delta / 2) \mathbf{c}_{\sigma}+i \sin (\Delta / 2) e^{i \Phi_{\mathrm{gcom}}} \mathbf{c}_{-\sigma}\right] .
$$

The sought after Babinet metasurface is ideally character ized by an effective $\sigma$ dependent contra circular complex transmittance,

$$
\tau_{\text {eff }} \propto \sin (\Delta / 2) \exp (2 i \sigma \psi) .
$$

Qualitatively, the proposed Babinet bilayered design shown in Fig. 1 in the one dimensional case of a square waveform with subwavelength spatial period $\Lambda$ can be understood from the fol lowing reasoning. Obviously, a slab of an isotropic dielectric is associated with a uniform dynamic phase. In contrast, once peri odically structured, it behaves as a uniaxial dielectric slab [11] with a dynamic phase that depends on the amount of the re moved material that is characterized by the filling factor $F$. The idea comes from the following intuition: the sequence of two complementary subwavelength gratings obtained by split ting an isotropic dielectric slab is thought as an effective subwave length structured slab without matter removal overall. As such, one could expect a constant dynamic phase independently of $F$, while the form birefringence remains strongly dependent on $F$.

The above reasoning is investigated quantitatively in the framework of the second order effective theory [12], by con sidering diamond material in air as a case study, whose refractive indices are, respectively, taken as $N=2.425$ and 1 at $\lambda=$ $532 \mathrm{~nm}$ wavelength. More precisely, a subwavelength grating illuminated at normal incidence and characterized with a filling factor $F$ and a period $\Lambda$ behaves as a uniaxial medium with refractive indices along a direction parallel $(\|)$ and perpendicular $(\perp)$ to the grating wavevector that are given by
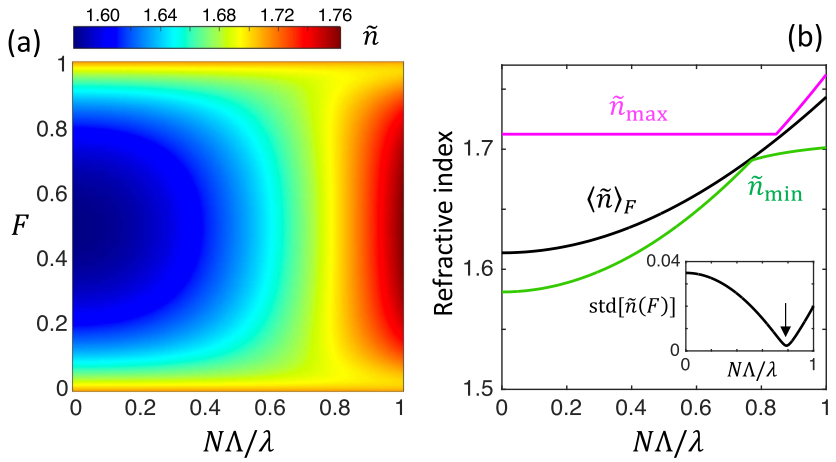

Fig. 2. (a) Effective average refractive index $\tilde{n}$ of the Babinet bilay ered subwavelength grating versus the reduced spatial period $N \Lambda / \lambda$ and the filling factor $F$. (b) Maximal $\left(\tilde{n}_{\max }=\max [\tilde{n}(F)]\right)$, minimal $\left(\tilde{n}_{\min }=\min [\tilde{n}(F)]\right)$, and average $\left(\langle\tilde{n}\rangle_{F}\right)$ effective refractive indices ver sus $N \Lambda / \lambda$. Inset: standard deviation of $\tilde{n}(F)$ as a function of the reduced period whose value $N \Lambda / \lambda=0.789$ (see arrow) defines the condition ensuring a minimal dependence of $\tilde{n}$ on $F$.

$$
\begin{gathered}
n_{\|}=n_{\|, 0}\left[1+\frac{\pi^{2} \Lambda^{2}}{3 \lambda^{2}} F^{2} \bar{F}^{2} n_{\|, 0}^{4} n_{\perp, 0}^{2}\left(\begin{array}{ll}
1 & \frac{1}{N^{2}}
\end{array}\right)^{2}\right]^{1 / 2}, \\
n_{\perp}=n_{\perp, 0}\left[1+\frac{\pi^{2} \Lambda^{2}}{3 \lambda^{2}} F^{2} \bar{F}^{2}\left(\begin{array}{ll}
N^{2} & 1
\end{array}\right)^{2}\right]^{1 / 2},
\end{gathered}
$$

where $\lambda$ is the wavelength, $\bar{F}=1 \quad F, n_{\perp, 0}=\left(N^{2} F+\bar{F}\right)^{1 / 2}$, and $n_{\|, 0}=N\left(F+N^{2} \bar{F}\right)^{-1 / 2}$.

Noting that the dynamic phase of the bilayer is $\Phi_{\mathrm{dyn}}=2 k L \tilde{n}$, where $k=2 \pi / \lambda$ and $L$ is the thickness of each layer and $\tilde{n}=(n+\bar{n}) / 2$ with $n$ and $\bar{n}$ being the average re fractive index of the first subwavelength grating and its comple ment, the dynamic phase behavior as a function of the structural characteristics is assessed via the study of $\tilde{n}$. This is reported in Fig. 2(a) where $\tilde{n}$ is plotted in the plane of param eters $(\Lambda, F)$. Here, the chosen range $0<N \Lambda / \lambda<1$ refers to the absence of diffraction orders other than the zeroth order one, which practically defines a subwavelength grating. Clearly, there is an optimal value for the grating period value that ensures a minimal dependence of $\tilde{n}$ on $F$, as illustrated in Fig. 2(b). Namely, as shown in the inset of that figure, $N \Lambda / \lambda=0.789$ minimizes the standard deviation of $\tilde{n}(F)$, which is the value used further in this work. The optical proper ties of the individual subwavelength gratings constituting the optimized bilayered element are summarized in Fig. 3, where the effective extraordinary $\left(n_{\|}\right.$and $\left.\bar{n}_{\|}\right)$, ordinary $\left(n_{\perp}\right.$ and $\left.\bar{n}_{\perp}\right)$, and average refractive $(n$ and $\bar{n}$ ) indices are plotted as a function of $F$. These two plots emphasize the compensation mechanism leading to an effective average refractive index of the bilayered optical element that varies much less with $F$ than the average refractive index of any of the two gratings taken separately. On the other hand, the birefringence of the complementary gratings have similar behavior, hence adding up.

Said differently, the filling factor can be used to modulate the birefringent phase retardation of the bilayer, $\Delta=4 \pi d \tilde{n} L / \lambda$ with $d \tilde{n}=\tilde{n}_{\|} \quad \tilde{n}_{\perp}$ and $\tilde{n}_{\|, \perp}=\left(n_{\|, \perp}+\bar{n}_{\|, \perp}\right) / 2$, without much variation of the dynamic phase. This is summarized in Fig. 4 where the effective average refractive index and bire fringence are plotted as a function of $F$. As shown in Fig. 4(a), $\tilde{n}$ exhibits slight modulations near $F \rightarrow 0$ and $F \rightarrow 1$, which implies that the optical element suffers from residual modula tion of the dynamic phase if one exploits the full range for $F$. Nevertheless, considering a situation that corresponds to a
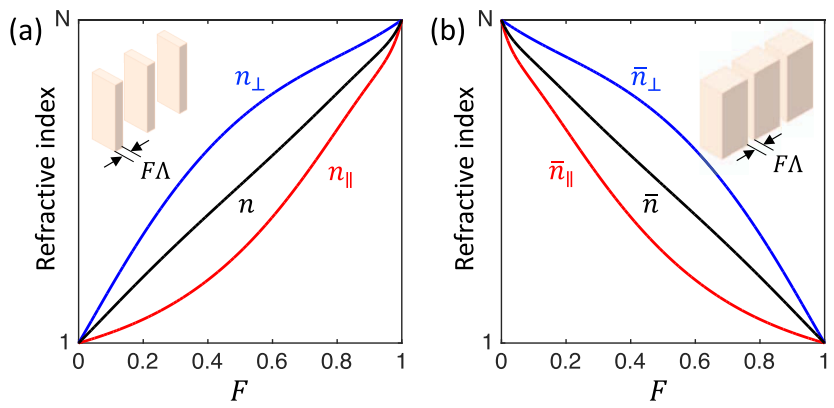

Fig. 3. (a) Extraordinary $\left(n_{\|}\right)$, ordinary $\left(n_{\perp}\right)$, and average $(n)$ refrac tive indices of a subwavelength grating as a function of the filling factor $F$. (b) Same as in (a) for the complementary subwavelength grating. The simulations are made for $N \Lambda / \lambda=0.789$, which optimizes the dynamic phase flatness. 
birefringent phase retardation $\Delta=\pi$ at $F=0.5$, there is a substantial decrease of the magnitude of the dynamic phase modulation in the case of the Babinet bilayer compared to the case of a single layer. This is illustrated in Fig. 5, where the variation of the dynamic phase in the range $0<F<0.5$ is shown in both cases, imposing a fixed maximal birefringent phase retardation at $F=0.5$. The dynamic phase profile is flat tened by a factor of $\simeq 42$ when considering the full range $0<F<0.5$, and this factor increases up to $\simeq 395$ by restrict ing to the range $0.2<F<0.5$, hence limiting the drawbacks associated with the resolution capabilities of nanofabrication tools as $F \rightarrow 0$. However, in order to obtain a full modulation of $\tau_{\text {eff }}$ between 0 and 1 , one has to increase $L$ in order to fulfill the condition $\Delta=2 \pi$ (i.e., $\tau_{\text {eff }}=0$ ) at $F=0.5$, which gives a thickness $L \simeq 450 \mathrm{~nm}$ and $\Delta=\pi$ (i.e., $\tau_{\text {eff }}=1$ ) at $F \simeq 0.17$ with the present parameters. Since the condition $N \Lambda / \lambda=$ 0.789 corresponds to $\Lambda \simeq 170 \mathrm{~nm}$, the latter conditions in volve the fabrication of subwavelength gratings with aspect ratio $L /(F \Lambda)$ up to 15 , which is accessible to nanofabrication techniques [13].

Next, the interest of the proposed concept is illustrated in the framework of beam shaping by considering the conception of geometric phase Laguerre Gaussian (LG) modal beam shapers. LG beams refer to a complete orthogonal basis for the scalar para xial Helmholtz equation [14], each element of the basis being univocally associated to an azimuthal index, $l$, and a radial index, $p$. Many strategies have been developed toward the generation of LG like beams using either extra cavity or intra cavity schemes, as reviewed in [9]. The use of geometric phase optical elements is especially interesting in that the helicity dependent geometric phase offers a route to switch between a mode $(l, p)$ and a mode $(l, p)$ by flipping the incident helicity of light from $\sigma$ to $\sigma$, provided that $\psi=(\sigma l / 2) \phi$.

Since the first LG like experimental demonstrations in the case $p=0$ in the early 2000s [15] and $p \geq 1$ only a few years ago $[16,17]$, the creation of geometric phase LG modal con verters as such has not yet been reported. Recently, a single layer theoretical proposal combining dynamic and geometric phase modulations at fixed $n_{\perp}$ has been reported [9]. However, the corresponding design breaks the right/left sym metry and thus works for only a single value of the helicity. A quasi modal version recovering the latter broken symmetry has been discussed on experimental grounds [18], though at the expense of substantial contra circular transmission losses.
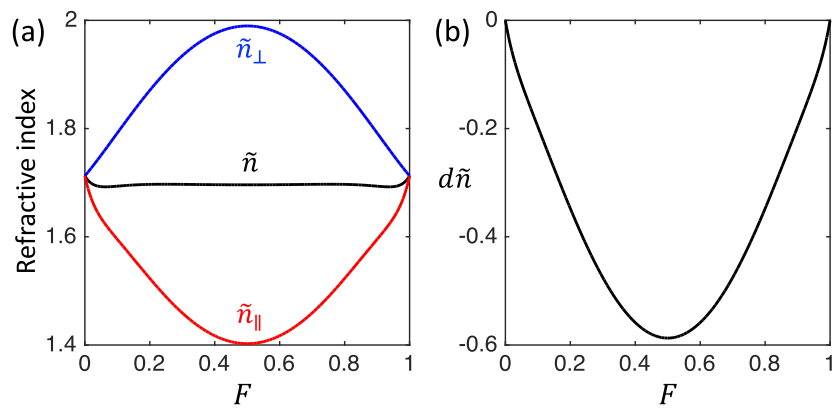

Fig. 4. (a) Extraordinary $\left(\tilde{n}_{\|}\right)$, ordinary $\left(\tilde{n}_{\perp}\right)$, and average $(\tilde{n})$ effective refractive indices of the optimized Babinet bilayered subwavelength grating that corresponds to $N \Lambda / \lambda=0.789$ according to the inset of Fig. 2(b). (b) Corresponding effective birefringence, $d \tilde{n}=\tilde{n}_{\|} \quad \tilde{n}_{\perp}$.
Here, we show that the use of space variant Babinet bilayered subwavelength gratings allows to create polarization controlled LG modal beam shapers. This is discussed in the representative case $p=0$; hence, the index $p$ is omitted in what follows, and we note that the generalization to high order radial modes poses no particular problem except more cumbersome calculations. At first, we recall that a $\mathrm{LG}_{l}$ field associated with the waist radius $w$ is expressed in its focal plane as

$$
E_{l}^{(w)} \propto\left(\frac{r}{w}\right)^{|l|} \exp \left(\frac{r^{2}}{w^{2}}+i l \phi\right) .
$$

Then we deduce from Eq. (2), which describes an ideal Babinet bilayer optical element, that the use of a birefringence phase retardation radial profile $\Delta_{l}(r)$ satisfying the condition

$$
\sin \left[\Delta_{l}(r) / 2\right]=\frac{r^{|l|} \exp \left(\frac{r^{2}}{w^{2}}+\frac{r^{2}}{w_{\text {in }}^{2}}\right)}{\max _{r}\left[r^{|l|} \exp \left(\frac{r^{2}}{w^{2}}+\frac{r^{2}}{w_{\text {in }}^{2}}\right)\right]},
$$

combined with an optical axis orientation angle

$$
\psi_{l}(\phi)=(l / 2) \phi \text {, }
$$

ensures the generation of a $\mathrm{LG}_{\sigma l}$ mode with waist $w$ by placing the optical element in the focal plane (or, in practice, by using a collimated beam) of a normally incident circularly polarized Gaussian beam with waist $w_{\text {in }}$, provided that $w<w_{\text {in }}$. The efficiency of the device (i.e., the fraction of the incident power that is converted into the desired mode power) is $\left(4 / w_{\text {in }}^{2}\right) \int_{0}^{\infty} \sin ^{2}\left[\Delta_{l}(r) / 2\right] \exp \left(2 r^{2} / w_{\text {in }}^{2}\right) r \mathrm{~d} r$ and depends on $w / w_{\text {in }}$. Its optimal value is reached for $w_{\text {opt }}=$ $w_{\text {in }}(1+|l|)^{-1 / 2}$. In this case, introducing $\rho=r / w_{\text {in }}$, Eq. (6) simplifies to

$$
\sin \left[\Delta_{l}^{\mathrm{opt}}(\rho) / 2\right]=2^{|l| / 2} \rho^{|l|} \exp \left(|l| \rho^{2}+|l| / 2\right) .
$$

The modal figure of merit of a space variant Babinet bilayer design given by Eqs. (7) and (8) is assessed quantitatively by evaluating the optimal power fraction $C_{\sigma l}$ of the $\sigma$ polarized field component at the output of the element that corresponds to a $\mathrm{LG}_{\sigma l}$ mode. Namely,

$$
C_{\sigma l}(w)=\frac{\left|\int_{0}^{\infty} E_{l}^{(w) *}\left[\mathbf{E}_{\text {out }} \cdot \mathbf{c}_{-\sigma}^{*}\right] r \mathrm{~d} r\right|^{2}}{\int_{0}^{\infty}\left|E_{l}^{(w)}\right|^{2} r \mathrm{~d} r \int_{0}^{\infty}\left|\left[\mathbf{E}_{\text {out }} \cdot \mathbf{c}_{-\sigma}^{*}\right]\right|^{2} r \mathrm{~d} r},
$$

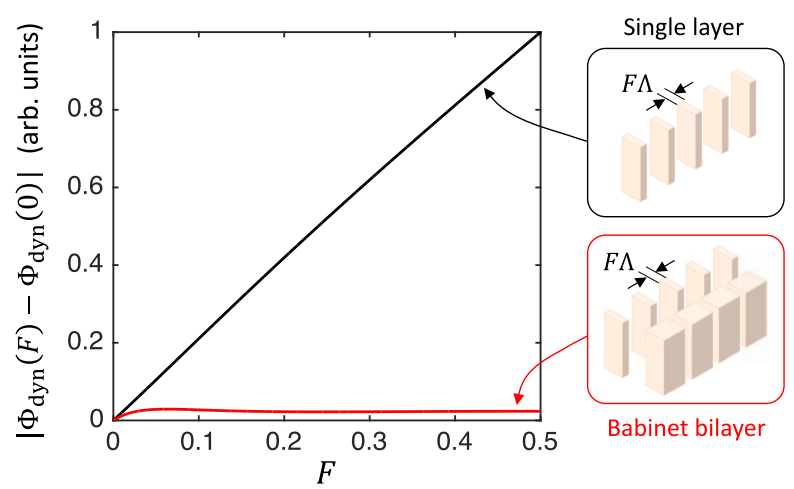

Fig. 5. Normalized variation of the dynamic phase as a function of the filling factor for a single subwavelength grating (black curve) and its comparison with the optimized Babinet bilayer (red curve) for a given maximal retardance at $F=0.5$. 


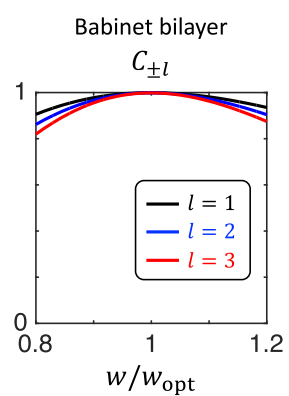

Non-twisted single layer
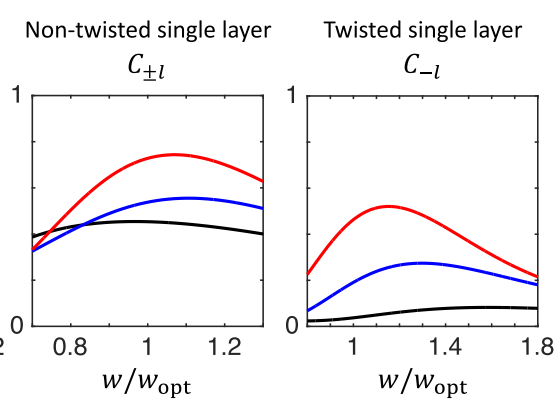

Fig. 6. Modal coefficients $C_{ \pm l}$ versus the waist $w$ of the sought after $\mathrm{LG}_{\sigma l}$ mode for three modal designs and $l=(1,2,3)$.

where $\mathbf{E}_{\text {out }}$ is given by Eq. (1), and the asterisk denotes complex conjugation, paying attention to the fact that LG modal decom position is not unique $[19,20]$. By considering the retardance range $0<\Delta<\pi$, we obtain a high degree of modality. Namely, $C_{ \pm l}>0.998$ for $l=(1,2,3)$, which is compared to two other situations. First, the case of single subwavelength gra tings characterized by the same structural features as the Babinet bilayers regarding both the optical axis orientation angle [Eq. (7)] and the birefringence phase retardation [Eq. (8)], to which we refer as the "non twisted single layer" case. Second, the case discussed in Ref. [9], to which we refer as the "twisted single layer" case, according to $\psi_{l}(r, \phi)=\left[\begin{array}{ll}l \phi & \Phi_{\mathrm{dyn}}(r)\end{array}\right] / 2$. In that case, the bire fringence phase retardation is also given by Eq. (8), while the op tical axis orientation angle is designed to cancel out the dynamic phase for one of the two helicity states (namely, $C_{+l}=1$ ) at the expense of doubling it for the other (namely, $C_{-l}<1$ ). The results are summarized in Fig. 6 and Table 1, both emphasiz ing the superior modal performances of Babinet bilayers.

In the field of optical information processing, the spin re versal symmetry of the proposed approach allows considering the extension of polarization controllled high order Poincaré spheres to the case of LG modes with given azimuthal and radial indices, $l$ and $p$. This solves a serious limitation of present approaches restricted so far to an infinite superposition of radial modes at fixed $l$, based on non modal geometric phase elements associated with $\psi=(l / 2) \phi$ and $\Delta=\pi$ [21].

In practice, there is no need for stringent subwavelength precision relative orientational alignment of the two subwave length gratings, as we are dealing with effective media. In addition, a monolithic design consisting of placing the two structured layers at both sides of a substrate can be considered, whose feasibility is supported by the previously reported fab rication of double sided geometric phase lenses [22]. Still, one should keep in mind that the grating depth and the nature of the surrounding media play important roles in the effective properties of the structured material [23]. In addition, Fresnel reflections may also be taken into account, at least for two main reasons: (i) the modification of the incident polarization as light enters into the structures due to optical anisotropy and (ii) cav ity effects associated with the presence of two structures. These possible drawbacks are evaluated at $F=0.5$. This gives for the case (i), the spin flipping of $\lesssim 10 \%$ of the incident photons for incident circular polarization, and for the case (ii), a typical Fabry Pérot finesse $\lesssim 1$. We conclude that although an attempt to implement such a design is not excluded in principle, quantitative attention must be kept in mind.
Table 1. Optimal Modal Coefficients $\max _{w}\left[C_{\sigma /}(w)\right]$ for Three Different Designs and $I=(1,2,3)$, which Corresponds to the Maximal Values Associated with the Plots Shown in Fig. 6

\begin{tabular}{lccc}
\hline Grating Type & $\boldsymbol{\ell}=\mathbf{1}$ & $\boldsymbol{\ell}=\mathbf{2}$ & $\boldsymbol{\ell}=\mathbf{3}$ \\
\hline Twisted monolayer $^{a}$ & $(1,0.082)$ & $(1,0.274)$ & $(1,0.520)$ \\
Non twisted monolayer $^{b}$ & 0.454 & 0.555 & 0.744 \\
Babinet bilayer $^{b}$ & 0.999 & 0.998 & 0.998 \\
\hline
\end{tabular}

"This case refers to Ref. [9], and the two values refer to $\sigma \quad \pm 1$.

${ }^{b}$ These cases are independent on $\sigma$.

Finally, we note that it is not the first time that Babinet's prin ciple has inspired the physics of metasurfaces and metamaterials, a pioneering example being the introduction of complementary split ring resonators acting as electric point dipoles with negative polarizability [24], which was followed by a huge number of de velopments. Present work therefore illustrates how basic optical concepts can bring attractive photonic opportunities to a field that has much progressed during the last two decades [25], in particular when the orbital angular momentum is at play.

\section{REFERENCES}

1. C. M. Soukoulis and M. Wegener, Nat. Photonics 5, 523 (2011).

2. M. Kadic, T. Bückmann, R. Schittny, and M. Wegener, Rep. Prog. Phys. 76, 126501 (2013).

3. K. Fan and W. J. Padilla, Mater. Today 18(1), 39 (2015).

4. A. M. Shaltout, A. V. Kildishev, and V. M. Shalaev, J. Opt. Soc. Am. B 33, 501 (2016).

5. G. Li, S. Zhang, and T. Zentgraf, Nat. Rev. Mater. 2, 17010 (2017).

6. A. E. Minovich, A. E. Miroshnichenko, A. Y. Bykov, T. V. Murzina, D. N. Neshev, and Y. S. Kivshar, Laser Photon. Rev. 9, 195 (2015).

7. Y. Guo, M. Pu, Z. Zhao, Y. Wang, J. Jin, P. Gao, X. Li, X. Ma, and X. Luo, ACS Photon. 3, 2022 (2016).

8. R. C. Devlin, A. Ambrosio, N. A. Rubin, J. B. Mueller, and F. Capasso, Science 358, 896 (2017).

9. M. Rafayelyan and E. Brasselet, Opt. Lett. 42, 1966 (2017).

10. E. Brasselet, Opt. Lett. 38, 3890 (2013). In that paper, " $q$ integer" should be read as " $q \in \mathbb{Z} / 2$."

11. D. C. Flanders, Appl. Phys. Lett. 42, 492 (1983).

12. S. Rytov, Sov. Phys. J. Exp. Theor. Phys. 2, 466 (1956).

13. M. Makita, P. Karvinen, V. A. Guzenko, N. Kujala, P. Vagovic, and C. David, Microelectron. Eng. 176, 75 (2017).

14. A. E. Siegman, Lasers (University Science Books, 1986).

15. G. Biener, A. Niv, V. Kleiner, and E. Hasman, Opt. Lett. 27, 1875 (2002).

16. P. Chen, B. Y. Wei, W. Ji, S. J. Ge, W. Hu, F. Xu, V. Chigrinov, and Y. Q. Lu, Photon. Res. 3, 133 (2015).

17. Y. He, Z. Liu, Y. Liu, J. Zhou, Y. Ke, H. Luo, and S. Wen, Opt. Lett. 40, 5506 (2015)

18. M. Rafayelyan, T. Gertus, and E. Brasselet, Appl. Phys. Lett. 110, 261108 (2017).

19. C. Schulze, S. Ngcobo, M. Duparré, and A. Forbes, Opt. Express 20, 27866 (2012).

20. G. Vallone, Opt. Lett. 42, 1097 (2017).

21. E. Karimi, S. Slussarenko, B. Piccirillo, L. Marrucci, and E. Santamato, Phys. Rev. A 81, 053813 (2010).

22. I. Mohacsi, I. Vartiainen, M. Guizar Sicairos, P. Karvinen, V. A. Guzenko, E. Müller, E. Färm, M. Ritala, C. M. Kewish, A. Somogyi, and C. David, Opt. Express 23, 776 (2015).

23. P. Lalanne and D. Lemercier Lalanne, J. Opt. Soc. Am. A 14, 450 (1997).

24. F. Falcone, T. Lopetegi, M. Laso, J. Baena, J. Bonache, M. Beruete, R. Marqués, F. Martn, and M. Sorolla, Phys. Rev. Lett. 93, 197401 (2004).

25. Y. Guo, M. Pu, X. Li, X. Ma, P. Gao, Y. Wang, and X. Luo, J. Phys. 30, 144003 (2018) 